\title{
Risk-Constrained Profit Maximization for Microgrid Aggregators with Demand Response
}

\author{
Duong Tung Nguyen and Long Bao Le, Senior Member, IEEE
}

\begin{abstract}
In this paper, we consider the operation optimization for a microgrid (MG) aggregator which can procure energy from various sources including the pool market and local distributed energy resources (DERs) to serve MG customers. We assume that the MG aggregator sells electricity to customers at a predefined retail rate and it also offers customers various contracts for adjusting their loads. Our design objective is to determine the optimal hourly bids that the MG aggregator submits to the dayahead (DA) market to maximize its profit. To deal with various uncertainties, a risk-constrained scenario-based stochastic programming framework is proposed where the MG aggregator's risk aversion is modeled using conditional value at risk (CVaR) method. The proposed formulation enables customers' demand response (DR) aggregation to be integrated into the operation of the MG aggregator via contractual agreements. This design is not only beneficial for both MG aggregator and customers, but also facilitates the operation of the system operator (SO) since a single entity (i.e., the MG aggregator) is visible to the SO instead of two separate entities (i.e., a MG aggregator and a DR aggregator). Extensive numerical results are shown to demonstrate the effectiveness of the proposed framework.
\end{abstract}

Index Terms-Demand response aggregation, conditional value at risk, two-stage stochastic optimization, microgrid aggregator.

\section{NOMENCLATURE}

Indices

$\begin{array}{ll}(.)_{., t, s} & \text { At time } t \text { in scenario } s \\ i, w, k & \text { Indices of DGs, WPs, and BESs } \\ r & \text { Indices LC/LS contracts } \\ t, s & \text { Indices of time slots and scenarios }\end{array}$

Parameters and Constants

$\beta \quad$ Risk-aversion parameter

$\Delta T \quad$ Duration of time slot (h)

$\eta_{k}^{\mathrm{C}}, \eta_{k}^{\mathrm{D}} \quad$ Charging/discharging efficiency of BES $k$

$c_{t}^{\mathrm{LL}} \quad$ Cost of involuntary load curtailment (\$/MWh)

$c_{w} \quad$ Cost of wind energy from WP $w(\$)$

$c_{r, t}^{\mathrm{LC}, 1}, c_{r, t}^{\mathrm{LS}, 1}$ Capacity cost of LC/LS contract $r(\$ / \mathrm{MW})$

$c_{r, t}^{\mathrm{LC}, 2}, c_{r, t}^{\mathrm{LS}, 2}$ Energy cost of LC/LS contract $r$ (\$/MW)

$e^{\mathrm{R}} \quad$ Electricity retail price $(\$ / \mathrm{MWh})$

$E_{k} \quad$ Capacity of BES $k$ (MWh)

$e_{t}^{\text {pen }} \quad$ Penalty for deviation between DA schedule and actual delivery (\$/MWh)

$N K, N G \quad$ Number of BESs and DGs

$N L C, N L S$ Number of LC/LS contracts

$N S, N T \quad$ Number of scenarios and time slots

NW Number of WPs

$p \quad$ DR price scaling factor

$P_{i}^{\min }, P_{i}^{\max }$ Min/max power generation of unit $i$ (MW)

$P_{t}^{\text {grid }} \quad$ Max exported/imported power (MW)

$P_{k}^{\mathrm{C}}, P_{k}^{\mathrm{D}} \quad$ Max charging/discharging power of BES $k(\mathrm{MW})$

Manuscript received January 18, 2014; revised April 12, 2014; accepted August 2, 2014

The authors are with INRS-EMT, Université du Québec, Montréal, Québec, Canada. Emails: \{duong.nguyen,long.le\}@emt.inrs.ca. L. B. Le is the corresponding author.

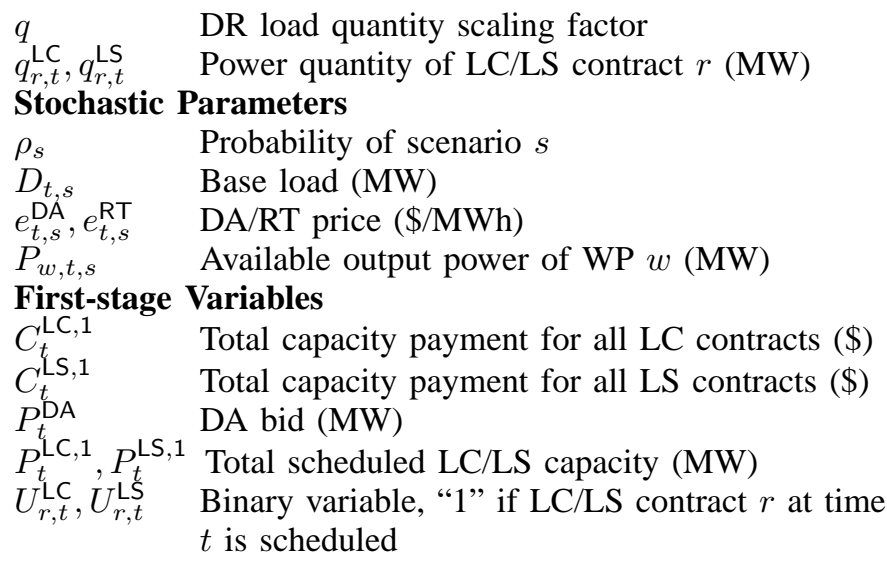

Second-stage Variables

$b_{k, t, s}^{\mathrm{C}}, b_{k, t, s}^{\mathrm{D}}$ Binary variable, " 1 " if charging/discharging

$C_{t C}^{\mathrm{LC}, 2}$

$C_{t, s}^{\mathrm{LC}, 2}$

$C_{t, s}^{\mathrm{L}, \mathrm{C}}, C_{t, s}^{\mathrm{LS}}$

Total energy payment for all LC contracts (\$)

$C_{t, s}^{\mathrm{LS}, 2}$

Total payment for all LC/LS contracts $(\$)$

$D_{t, s}^{\mathrm{ILCC}}$

Total energy payment for all LS contracts (\$)

$I_{i, t, s} \quad$ Commitment status of DG $i\{0,1\}$

$P_{i, t, s} \quad$ Power generation of DG $i$ (MW)

$P_{k, t, s}^{C}, P_{k, t, s}^{\mathrm{D}}$ Charging/discharging power of BES $k$ (MW)

$P_{t, s}^{\text {del }} \quad$ Actual power delivery (MW)

$P_{t, s}^{\mathrm{LC}, 2}, P_{t, s}^{\mathrm{LS}, 2}$ Total LC/LS deployment (MW)

$P_{w, t, s}^{\text {ws }} \quad$ Power curtailment of WP $w(\mathrm{MW})$

$S O C_{k, t, s} \quad$ SOC of BES $k$

$S U_{i, t, s} \quad$ Startup cost of DG $i(\$)$

$u_{r, t, s}^{\mathrm{LC}}, u_{r, t, s}^{\mathrm{LS}}$ Binary variable, " 1 " if LC/LS contract $r$ at time $t$ is deployed in scenario $s$

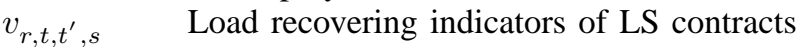

$y_{i, t, s}, z_{i, t, s} \quad$ Startup and shutdown indicators of DG $i$

\section{INTRODUCTION}

Demand response (DR) is a vital component in the future smart grids, which can be employed to facilitate the integration of intermittent and variable energy sources such as wind or solar energy into the power system, and to ensure the balance between demand and supply. DR also has great potential to reduce peak load, which helps defer the need of generation capacity expansion as well as reduce the system operation cost and carbon footprint by operating a smaller number of generators to meet the peak load. In fact, after the release of Order 745 issued by Federal Energy Regulatory Commission (FERC) [1], several DR programs have been introduced by system operators to encourage participation of various DR sources in the power system [2], [3].

Research on DR issues has received lots of interests in recent years. References [4]-[9] proposed various market clearing models considering the DR integration. In [10], the authors presented a real-time DR model for customers 
in response to time-varying electricity price using robust optimization techniques. Chen et al. [11] employed online stochastic programming and robust optimization methods to minimize the total energy cost for residential household with flexible loads. A novel optimization framework was introduced in [12] to maximize the profit of a DR aggregator in the day-ahead (DA) energy market. Furthermore, various load reduction strategies were proposed in [12].

Microgrid (MG) is another important concept in the smart grids. A MG can be defined as a cluster of distributed energy resources (DERs) and associated load where it can maintain the operation while being disconnected from the main grid in certain circumstances. Deployment of MGs can facilitate efficient demand side management and integration of renewable energy sources (RESs) at the distribution level. MG research has drawn a great deal of attention from the research community recently. In [13]-[20], the authors proposed various models to optimize the operation of MGs. In most existing works, the MG aggregator is considered as a non-profit agent, which can fully control local DERs and load. Major design goals in these existing works are either to minimize the operating cost for the whole MG or to balance the local energy generation and demand. The MG aggregator can also participate in the energy market and sell electricity to customers [21]. In [22], the authors introduced a framework for co-optimization of generation and transmission planning considering the installation aspects of MGs.

In this paper, we focus on the operation optimization of a MG aggregator that trades energy in the pool market and procures energy from local DERs to serve MG customers. Our design objective is to determine the optimal bidding strategy for the MG aggregator in the DA energy market and schedule the energy dispatch in real time to maximize the profit of the aggregator. In addition, the concept of DR aggregation contracts [12] between the MG aggregator and customers is integrated into the bidding decision making process of the aggregator. Our design enables the $\mathrm{MG}$ aggregator to exploit the flexibility of the load to mitigate the negative impacts of the uncertainties due to renewable energy generation and electricity prices, which, consequently, increases the profit. Moreover, MG customers can reduce their bills by receiving rewards from the MG aggregator for their DR capabilities. In our proposed framework, the $\mathrm{MG}$ aggregator and $\mathrm{DR}$ aggregator are merged into one single entity, which would ease the operation of the SO.

The power scheduling and bidding problem for the MG aggregator is formulated as a two-stage scenario-based stochastic program which accounts for various uncertainties in the system. Furthermore, risk control is embedded in the proposed optimization framework by employing the conditional value at risk (CVaR) approach [23]-[25]. Indeed, CVaR is a wellknown risk measure that has been widely used in various energy management problems for different entities in the electricity market such as retailers [26]-[28], producers [29][32], distribution companies [33], energy customers [34], and in generation operation planning [35], optimal power flow [36] as well as coordinated energy trading problems [37], [38]. The main contributions of this paper can be summarized as follows:

- We introduce a comprehensive model for power scheduling and bidding operation of a MG aggregator in the deregulated electricity market considering RESs and DR. The proposed model is general, which can be applied to other entities such as a distribution company (DISCO), a retailer, or a load serving entity (LSE) [24] where these entities could act as a MG aggregator.

- We present a novel DR settlement scheme between the MG aggregator and MG customers. A similar principle to generation unit commitment (UC) concept in DA market clearing problem is utilized by the MG aggregator to schedule DR contracts. A risk-constrained twostage stochastic programming formulation is proposed to represent the underlying optimization problem where the risk aversion of the $\mathrm{MG}$ aggregator is captured by using the conditional value at risk (CVaR) approach.

- Extensive simulation results are presented to illustrate the great benefits of establishing DR contracts between the MG aggregator and MG customers. Also, the impacts of different system and design parameters on the optimal solution are evaluated via the sensitivity analysis.

The remainder of this paper is organized as follows. Section II describes the system model and the solution approach. Detailed problem formulation is presented in Section III. Several case studies and numerical results are provided in Section IV. Finally, Section V states the concluding remarks and discusses some directions for future works.

\section{System Model AND Modeling ApproACH}

\section{A. System Model}

We consider a large-scale MG (i.e., average hourly load is in the range of several $\mathrm{MW}$ ), which could represent a residential community or a large university campus for example. The MG aggregator sells electricity to MG customers at a fixed retail rate, which is based on the agreement between the aggregator and its customers, and it can also procure energy from various energy sources including local DERs and the wholesale electricity market. The DERs can be renewable sources, non-renewable sources, and BESs. The MG aggregator itself may own several wind power units (WPs) or it may have take-or-pay contracts ${ }^{1}$ to buy wind energy from local wind farms [13]. In a take-or-pay contract, we make the same assumptions as in [13] where the MG aggregator agrees to buy all available wind energy generation from wind farms. The wind energy price that the MG aggregator pays to wind farms is typically lower than the electricity price from the energy market. Furthermore, we assume the MG aggregator possesses a few BESs and several DGs such as microturbines, fuel cells, and diesel generators.

The MG aggregator can also procure extra energy from the wholesale market. Additionally, the MG aggregator is allowed to sell energy back to the market when the local generation is surplus (e.g., during high wind periods). We assume the MG aggregator is a price-taker; thus, it needs to submit only the hourly power bid quantities to the DA market, and the revenue (cost) obtained from selling (buying) electricity in the market is settled at the Market Clearing Price [24], [39]-[41].

The operation of the MG in the energy market is summarized as follows. The MG aggregator submits the hourly power quantities that it commits to buy/sell in the DA market to the market operator (MO) several hours before the operating day. For example, in PJM DA energy market [2], selling/buying bids must be submitted to the MO by 12 P.M. of the day before. During the operating day, depending on

${ }^{1}$ In some markets, it is called Power Purchase Agreements (PPAs). 
actual conditions of the system (e.g., actual wind generation and load), the MG aggregator may participate in the real-time (RT) energy market to compensate for the deviation from the DA schedule. The deviation between the DA schedule and the actual power delivery may be subject to an energy imbalance charge imposed by MO [24], [30], [39]-[42]. Additionally, the MG aggregator also needs to make decisions on battery charging/discharging, unit commitment, energy dispatch for DGs, and wind energy dispatch over the operating day.

We assume that the MG aggregator offers DR programs including load curtailment (LC) and load shifting (LS) contracts to MG customers. The MG aggregator itself can directly communicate with customers or it can make these LC/LS contracts available through an intermediate agent (e.g., a DR aggregation agent) to collect the aggregated DR offers from MG customers. The MG aggregator rewards customers who participate in the DR programs through two types of payments, namely capacity payment and energy payment [9]. If a LC/LS contract is scheduled ${ }^{2}$, it will receive a capacity payment based on the scheduled LC/LS quantity in the contract. If a LC/LS contract is both scheduled and deployed in the operating day, it will receive an additional energy payment. Each LC contract includes the LC quantity, the capacity price, and the energy price for each time slot during the operating day [12]. Each LS contract includes the LS quantity, the capacity price, the energy price, and the set of time slots to which the offered load in the LS contract can be shifted $[12]^{3}$. Detailed LC contracts and LS contracts will be further discussed in Sections III-A7 and III-A8, respectively.

\section{B. Stochastic Optimization Approach}

The MG aggregator faces various uncertainties in system load, wind energy generation, and DA and RT electricity prices when it makes the DA bidding decision. To deal with these uncertainties, the two-stage scenario-based stochastic programming approach is employed [24]. Specifically, we employ the Monte Carlo simulation with Latin Hypercube Sampling (LHS) technique [43], [44] to generate a large number of scenarios representing the uncertain parameters based on their corresponding distribution functions [24], [37]. Each scenario captures the information of the hourly load, the hourly wind speed, and the DA and RT electricity prices in the operating day. Wind power can be calculated from wind speed based on the wind power curve. We employ the piecewise linear approximation of the wind power curve as follows [45]:

$$
P= \begin{cases}0, & \text { if } v \leq v^{\mathrm{ci}} \text { or } v \geq v^{\mathrm{co}}, \\ P^{\mathrm{r}} \frac{v-v^{\mathrm{ci}}}{v^{\mathrm{r}}-v^{\mathrm{ci}}}, & \text { if } v^{\mathrm{ci}} \leq v \leq v^{\mathrm{r}}, \\ P^{\mathrm{r}}, & \text { otherwise }\end{cases}
$$

where $P$ is the available wind power generation, $P^{r}$ is the rated power of the wind turbine, $v$ is the wind speed, and $v^{\mathrm{ci}}, v^{\mathrm{co}}$, and $v^{\mathrm{r}}$ are the cut-in, cut-out, and rated wind speeds, respectively.

The scenario generation process can be implemented as follows. We assume that the MG aggregator can obtain forecast data from a local forecasting center or the MG aggregator itself has suitable tools to forecast the system uncertainty factors

\footnotetext{
${ }^{2}$ If a LC/LS contract is scheduled, it may or may not be deployed in the operating day.

${ }^{3}$ We assume that the task of reallocating/recovering LS load is performed by the MG aggregator instead of the ISO operator as in [12].
}

with a high degree of accuracy. State-of-the-art forecasting techniques such as time series prediction, artificial neural networks, and support vector machines can be utilized for forecasting the wind speed, electricity prices, and load [24]. In other words, we suppose that the forecasts for uncertainties in the considered system model can be achieved, whose detailed design is outside the scope of this paper.

Any forecasting techniques can suffer from forecasting errors and modeling such forecasting errors is an important research topic itself, which is not the focus of this paper. In addition, an efficient model for the forecasting errors can also depend on the considered data sets. To enable the considered MG operation optimization design, we assume that the MG aggregator can fit the forecasting error data into appropriate distributions or time series based on the historical actual data and forecast data over a sufficiently long period (e.g., one year). For simplicity, the forecasting errors of wind speed, system load, DA and RT electricity prices are assumed to follow normal distributions with zero-mean and the standard deviations are 5\% [17], $10 \%, 20 \%$ [18], and $25 \%$ of their corresponding forecast values. The standard deviation for RT price is set to be higher than that for DA price to reflect a typical scenario in practice [39]. Furthermore, we assume that the system uncertainties are independent [9]. Modeling the correlation among the uncertain parameters is also beyond the scope of this paper. Note that the assumption that forecasting errors of system load, wind, and electricity prices follow normal distributions can be found in many previous works [9], [11], [15], [17], [18], [37], [39], [41], [43], [46]. However, other distributions (e.g., Weibull distribution for wind speed) or other scenario generation methods can also be used for our proposed optimization framework.

Based on the distributions of uncertainty parameters, 5000 scenarios with even probability (1/5000) are generated by using the Monte Carlo simulation with LHS technique. On one hand, it is desirable to generate a larger number of scenarios to reliably capture the underlying system uncertainty factors. However, the number of generated scenarios directly impacts the computation complexity of the proposed scenario-based stochastic optimization problem. On the other hand, a too small number of scenarios may reduce the accuracy of the results. Therefore, the tradeoff between computational complexity and modeling accuracy needs to be considered. Guided by some in-sample and out-of-sample stability tests, we have decided to select 100 scenarios in the reduced scenario tree.

The key idea in scenario reduction is to eliminate scenarios with very low probabilities and to aggregate scenarios of close distances based on certain probability metric [43], [47], [48]. The outputs of a scenario reduction algorithm are a set of a smaller number of scenarios and their corresponding probabilities. We used GAMS/SCENRED package [50], [51] to implement the scenario reduction process. Due to its computational advantage, the fast-forward scenario reduction technique [24], [34], [47]-[49] is utilized to reduce the original 5000 scenarios to 100 scenarios. In the fast-forward reduction algorithm, the reduced scenario tree is built by selecting/adding one scenario from the original tree at each iteration. Therefore, this algorithm has low computation time, which is critical since we have to process a very large number of scenarios to attain a small number of scenarios (e.g., from 5000 scenarios to 100 scenarios in this paper) [24], [47]-[49]. All simulations required to obtain numerical results in Section 
IV are implemented on a desktop computer with $3.5 \mathrm{GHz}$ Intel Core i7-3370 CPU and 16GB RAM. The computation time needed for reducing from 5000 scenarios to 100 scenarios using GAMS/SCENRED is less than 35 seconds.

\section{Risk Management}

The actual profit of the MG aggregator in the proposed stochastic optimization model is indeed a random variable [24]. In the risk-neutral formulation, we solely maximize the expected value of the profit while ignoring the remaining parameters characterizing the distribution of the profit [23], [24], [49]. However, the achieved optimal expected profit may come with a high level of profit variability and entail a high possibility of low profits or even negative profits (losses). A risk management scheme is typically desired to control of outcome volatility and avoid non-desirable profit distributions due to various system uncertainties.

Several risk measures (e.g., variance, shortfall probability, expected shortage, value-at-risk, conditional value at risk, stochastic dominance) have been proposed in the literature to control the trade-off between expected profit and the variability of such profit [24]. In this paper, the MG aggregator's risk aversion is modeled by using the conditional value at risk (CVaR) method due to its advantage and popularity over other risk measures available in the literature [24]. Rich literature on CVaR methodology can be found in [23]-[38].

The conditional value at risk at $\alpha$ confidence level ( $\alpha$-CVaR) can be defined as the expected value of the profit smaller than the (1- $\alpha)$-quantile of the profit distribution. In other words, in the scenario-based stochastic optimization method, $\alpha$-CVaR is defined approximately as the expected profit in the $(1-\alpha) \mathrm{x}$ $100 \%$ worst scenarios [24], [30]. The (1- $\alpha)$-quantile of the profit distribution is known as Value-at-Risk (VaR), which is the largest value ensuring that the probability of obtaining a profit less than that value is lower than 1- $\alpha, \forall \alpha \in(0,1)$. The $\alpha-\mathrm{CVaR}$ is the optimal value of the objective function in the following optimization problem [23], [24], [26], [27], [30]:

$$
\max _{\xi, \eta_{s}} \quad \xi-\frac{1}{1-\alpha} \sum_{s=1}^{N S} \rho_{s} \eta_{s}
$$

subject to

$$
\begin{aligned}
\eta_{s} \geq \xi-\text { profit }_{s}, & \forall s \\
\eta_{s} \geq 0, & \forall s
\end{aligned}
$$

where $\alpha$ is the confidence level, profit $t_{s}$ is the profit in scenario $s, \rho_{s}$ is probability of scenario $s, \xi$ presents $\mathrm{VaR}, \eta_{s}$ is an auxiliary non-negative variable equal to the difference between VaR and profit s $_{s}$ profit ${ }_{s}$ is smaller than VaR, and equal to zero otherwise. Intuitively, $\eta_{s}$ measures the distance between $\mathrm{VaR}$ and the profit in scenario $s$ when the profit in scenario $s$ is smaller than VaR.

When CVaR is considered, we attempt to maximize the sum of the expected profit and the CVaR value of the profit multiplied by a weighting parameter (i.e., risk aversion parameter $\beta$ ) instead of maximizing the expected profit alone. The CVaR at $\alpha$ confidence level can be integrated into the risk-neutral problem by modifying the objective function as follows [24], [26], [30], [37]:

$$
\max \sum_{s=1}^{N S} \rho_{s} \text { Profit }_{s}+\beta C V a R
$$

where

$$
C V a R=\xi-\frac{1}{1-\alpha} \sum_{s=1}^{N S} \rho_{s} \eta_{s} .
$$

Furthermore, the constrains (3)-(4) must be added to the set of constraints in the original risk-neutral optimization problem. Here, $\beta$ is the risk-aversion parameter. When $\beta$ is equal to zero (i.e., risk control is not considered), the $\mathrm{MG}$ aggregator is a risk-neutral decision maker. Moreover, the $\mathrm{MG}$ aggregator becomes more risk-averse as $\beta$ increases.

\section{Implementation Issues}

The proposed optimization framework can be implemented as follows. In the day before the operating day, the MG aggregator collects all the LC/LS contract information (e.g., from a DR aggregation agent), forecasts system uncertainties including system load, wind generation, and DA and RT electricity prices over the operating day, then runs the scenario generation and scenario reduction algorithms. The inputs to the underlying optimization problem, which will be presented in Section III, include LC/LS contract information, specification parameters of system components such as BESs and DGs, and a set of scenarios representing the system uncertainties.

When all these inputs are available, the MG aggregator calculates the optimal hourly energy bids for buying/selling electricity in the DA market and submits the optimal bidding decision to the market operator before a predefined bidding deadline (e.g., 12 P.M. of the day before the operating day). Also, the MG aggregator computes the optimal scheduled LC/LS quantities in DR contracts ${ }^{4}$. Customers with scheduled LC/LS contracts will receive capacity payments which are proportional to the optimal scheduled LC/LS quantities. The optimal DA bidding decision and the optimal scheduled DR capacity decision are the first-stage decision variables in the proposed two-stage stochastic optimization model. These decisions must be made before the system uncertainties are unveiled considering possible realizations of uncertainties at the second stage.

In addition to first-stage decisions, the outputs of the optimization problem contain second-stage decisions (recourse decisions) which are made after the uncertainties are disclosed, and they depend on the first-stage decisions. Each scenario (i.e., one realization of the system uncertainties) corresponds to a set of second-stage decisions which include the actual power delivery between the MG and the main grid, the operation of DGs, charging activities of BESs, decisions on actual deployment of LC/LS contracts, and involuntary load curtailment as well as wind energy curtailment.

In summary, the MG operation optimization is formulated as a two-stage stochastic program where the first-stage decisions include the hourly power quantities that the MG aggregator submits to the DA market, and the commitment statuses of LC and LS contracts while the second-stage decisions are the actual power delivery, the operation of DGs, charging/discharging decisions of BESs, the deployment of LC and LS contracts, and the amount of involuntary load curtailment and wind energy curtailment. The outcomes of the optimization problem include both first-stage and second-stage decisions.

\footnotetext{
${ }^{4}$ These decisions correspond to commitment statuses of LC and LS contracts.
} 


\section{PROBLEM Formulation}

\section{A. Risk-Neutral Stochastic Optimization Formulation}

In the risk-neutral formulation, the objective of the $\mathrm{MG}$ aggregator is solely to maximize its expected profit. The objective and constraints of the risk-neutral problem are presented in the following.

1) Objective Function: We are interested in maximizing the expected profit of the MG aggregator as follows:

$$
\max \sum_{s=1}^{N S} \rho_{s} \text { Profit }_{s}
$$

where the profit of the MG aggregator in scenario $s$ is

$$
\begin{gathered}
\text { Profit }_{s}=\sum_{t=1}^{N T}\left\{\Delta T \left[e^{\mathrm{R}}\left(D_{t, s}-P_{t, s}^{\mathrm{LC}, 2}-D_{t, s}^{\mathrm{LC}}\right)+P_{t}^{\mathrm{DA}} e_{t, s}^{\mathrm{DA}}\right.\right. \\
+\left(P_{t, s}^{\mathrm{del}}-P_{t}^{\mathrm{DA}}\right) e_{t, s}^{\mathrm{RT}}-e_{t}^{\mathrm{pen}}\left|P_{t, s}^{\mathrm{del}}-P_{t}^{\mathrm{DA}}\right|-\sum_{w=1}^{N W} c_{w} P_{w, t, s} \\
\left.\left.-c_{t}^{L L} D_{t, s}^{\mathrm{ILC}}\right]-\left(C_{t, s}^{\mathrm{LC}}+C_{t, s}^{\mathrm{LS}}\right)-\sum_{i=1}^{N G}\left[C\left(P_{i, t, s}\right)+S U_{i, t, s}\right]\right\} .
\end{gathered}
$$

The objective function is the sum of the revenues obtained from selling electricity to the MG customers and trading energy in electricity markets, minus the operating cost of WPs (or the cost of purchasing energy from WPs), the involuntary load curtailment penalty, the payments for LC and LS contracts, the operating cost, and start-up cost of DGs. Note that the operating cost of a WP is typically small. Therefore, for a MG aggregator-owned WP $w$, we can set $c_{w}$ to 0 ; and for any WP $w$ which is not owned by the MG aggregator, $c_{w}$ is set equal to a positive value representing the price that $\mathrm{MG}$ aggregator agreed to buy energy generated from WP $w$.

In the following, equation (9) represents the expected revenue of the MG aggregator due to selling electricity at fixed retail rate $\left(e_{t}^{R}\right)$ to $\mathrm{MG}$ customers. In scenario $s$, the total original load over the scheduling day is $\Delta T \sum_{t=1}^{N T} D_{t, s}$, and the total amount of load curtailment is $\Delta T \sum_{t=1}^{N T}\left(P_{t, s}^{\mathrm{LC}, 2}+D_{t, s}^{\mathrm{ILC}}\right)$ where $P_{t, s}^{\mathrm{LC}, 2}$ is the total $\mathrm{LC}$ amount from all LC contracts at time $t$. The net revenue of the MG aggregator via trading in the energy market is given in (10), which is equal to the revenue obtained from selling/buying energy in DA and RT markets minus the energy imbalance charge for the deviation between the DA schedule and the RT power delivery [30], [39], [42]. Also, the power traded between the MG aggregator and the main grid in RT market at time $t$ in scenario $s$ is $P_{t, s}^{\text {del }}-P_{t}^{\mathrm{DA}}$.

$$
\begin{array}{r}
R e v^{\text {retail }}=\sum_{s=1}^{N S} \rho_{s} \sum_{t=1}^{N T} \Delta T\left(e^{\mathrm{R}} D_{t, s}-e^{\mathrm{R}} P_{t, s}^{\mathrm{LC}, 2}-e^{\mathrm{R}} D_{t, s}^{\mathrm{ILC}}\right) \\
R e v^{\text {market }}=\sum_{s=1}^{N S} \rho_{s} \sum_{t=1}^{N T} \Delta T\left(P_{t}^{\mathrm{DA}} e_{t, s}^{\mathrm{DA}}+\left(P_{t, s}^{\text {del }}-P_{t}^{\mathrm{DA}}\right) e_{t, s}^{\mathrm{RT}}\right. \\
\left.-e_{t}^{\text {pen }}\left|P_{t, s}^{\mathrm{del}}-P_{t}^{\mathrm{DA}}\right|\right) .
\end{array}
$$

The system constraints are given in the following.

2) Power Exchange with Main Grid: We must have

$$
-P_{t}^{\text {grid }} \leq P_{t, s}^{\text {del }} \leq P_{t}^{\text {grid }}, \forall t, s ; \quad-P_{t}^{\text {grid }} \leq P_{t}^{\mathrm{DA}} \leq P_{t}^{\text {grid }}, \forall t
$$

3) Operation Constraints for DGs: For $\forall i, t, s$, we have the following constraints

$$
\begin{array}{r}
C\left(P_{i, t, s}\right)=a_{i} I_{i, t, s}+\Delta T \sum_{m=1}^{N_{i}} \lambda_{i, m} P_{i, m, t, s} \\
0 \leq P_{i, m, t, s} \leq P_{i, m} ; \quad P_{i, t, s}=P_{i}^{\min } I_{i, t, s}+\sum_{m=1}^{N_{i}} P_{i, m, t, s} \\
P_{i}^{\min } I_{i, t, s} \leq P_{i, t, s} \leq P_{i}^{\max } I_{i, t, s} ; \quad S U_{i, t, s}=C U_{i} y_{i, t, s} \\
P_{i, t, s}-P_{i, t-1, s} \leq U R_{i}\left(1-y_{i, t, s}\right)+P_{i}^{\min } y_{i, t, s} \\
P_{i, t-1, s}-P_{i, t, s} \leq D R_{i}\left(1-z_{i, t, s}\right)+P_{i}^{\min } z_{i, t, s} \\
\sum_{h=t}^{t+U T_{i}-1}\left(1-I_{i, h, s}\right) \geq D T_{k} z_{i, t, s} \\
\sum_{h=t, t, s}-z_{i, t, s}=I_{i, t, s}-I_{i, t} \geq U T_{i} y_{i, t, s} ; \quad y_{i, t, s}+z_{i, t, s} \leq 1 .
\end{array}
$$

Production cost C(.) of DGs is approximated by piecewise linear functions [52], [53] where $m$ and $N_{i}$ denote the indices

of segments and number of segments in the cost function of DG $i$, respectively. The marginal cost associated with segment $m$ of DG $i$ cost function is $\lambda_{i, m}(\$ / \mathrm{MWh})$, and $a_{i}$ is the cost of running DG $i$ at its minimum power generation [52]. Additionally, $P_{i, m}$ is the upper limit of power generation from the $m$-th segment of DG $i$ cost function (MW) and $P_{i, m, t, s}$ is power generation of DG $i$ from the $m$-th segment at time $t$ in scenario $s$. Constraints (12)-(13) capture the generation cost and power output of DGs. Start-up cost and power generation limits are given in (14). The remaining constraints present limits on ramping rates, minimum ON/OFF duration, and relationship between binary variables [52], [53]. Note that $D R_{i} / U R_{i}$ is the ramping-down/up rate limit of unit $i$ and $D T_{i} / U T_{i}$ is the minimum down/up time of unit $i$.

4) Battery Constraints: For $\forall k, t, s$, we must have

$$
\begin{array}{r}
0 \leq P_{k, t, s}^{\mathrm{C}} \leq b_{k, t, s}^{\mathrm{C}} P_{k}^{\mathrm{C}} ; \quad 0 \leq P_{k, t, s}^{\mathrm{D}} \leq b_{k, t, s}^{\mathrm{D}} P_{k}^{\mathrm{D}} \\
S O C_{k, N T, s}=S O C_{k, 1} ; S O C_{k}^{\mathrm{min}} \leq S O C_{k, t, s} \leq S O C_{k}^{\max } \\
S O C_{k, t+1, s}=S O C_{k, t, s}+\Delta T\left(\frac{\eta_{k}^{\mathrm{C}} P_{k, t, s}^{\mathrm{C}}}{E_{k}}-\frac{P_{k, t, s}^{\mathrm{D}}}{\eta_{k}^{\mathrm{D}} E_{k}}\right) \\
b_{k, t, s}^{\mathrm{C}}+b_{k, t, s}^{\mathrm{D}}=1 ; \quad b_{k, t, s}^{\mathrm{C}}, b_{k, t, s}^{\mathrm{D}} \in\{0,1\} .
\end{array}
$$

Constraints (20)-(21) impose limits on the charging and discharging power as well as limits on battery State of Charge (SOC). $\mathrm{SOC}_{k}^{\text {max }}$ and $\mathrm{SOC}_{k}^{\min }$ are the maximum and minimum SOC of battery $k$, respectively. Battery energy dynamic model is described in (22) while constraint (23) captures conditions on binary variables representing battery charging/discharging status.

5) Involuntary Load Curtailment: In every scenario, the amount of ILC is always smaller than the base load, i.e.,

$$
0 \leq D_{t, s}^{\mathrm{ILC}} \leq D_{t, s}, \quad \forall t, s .
$$

6) Renewable Energy Curtailment: Obviously, the amount of wind power curtailment must always be smaller than the available wind power generation, i.e.,

$$
0 \leq P_{w, t, s}^{w s} \leq P_{w, t, s}, \quad \forall w, t, s .
$$


7) Load Curtailment: Each LC contract $r$ includes the information of LC capacity price $\left(c_{r, t}^{\mathrm{LC}, 1}\right)$, LC energy price $\left(c_{r, t}^{\mathrm{LC}, 2}\right)$, and the aggregated LC quantity at time slot $t\left(q_{r, t}^{\mathrm{LC}}\right)$ from the customers [12]. Similar to [9], we consider two kinds of payments of the MG aggregator for LC participants, namely capacity payment and energy payment. If a LC contract $r$ is scheduled to be possibly curtailed at time $t$ in the operating day, it will receive a capacity payment. If a LC contract $r$ is scheduled at time $t$ (i.e., $U_{r, t}=1$ ) and it is actually curtailed (i.e., $u_{r, t, s}^{\mathrm{LC}}=1$ ), it will receive an additional energy payment. For $\forall r, t, s$, we have the following constraints

$$
\begin{array}{r}
P_{t}^{\mathrm{LC}, 1}=\sum_{r=1}^{N L C} q_{r, t}^{\mathrm{LC}} U_{r, t}^{\mathrm{LC}} ; \quad P_{t, s}^{\mathrm{LC}, 2}=\sum_{r=1}^{N L C} q_{r, t}^{\mathrm{LC}} u_{r, t, s}^{\mathrm{LC}} \\
C_{t}^{\mathrm{LC}, 1}=\sum_{r=1}^{\mathrm{NLC}} c_{r, t}^{\mathrm{LC}, 1} q_{r, t}^{\mathrm{LC}} U_{r, t}^{\mathrm{LC}} ; \quad C_{t, s}^{\mathrm{LC}, 2}=\sum_{r=1}^{\mathrm{NLC}} c_{r, t}^{\mathrm{LC}, 2} q_{r, t}^{\mathrm{LC}} u_{r, t, s}^{\mathrm{LC}} \\
C_{t, s}^{\mathrm{LS}}=C_{t}^{\mathrm{LS}, 1}+C_{t, s}^{\mathrm{LS}, 2} ; u_{r, t, s}^{\mathrm{LC}} \leq U_{r, t}^{\mathrm{LC}} ; \quad U_{r, t}^{\mathrm{LC}}, u_{r, t, s}^{\mathrm{LC}} \in\{0,1\} .
\end{array}
$$

The total scheduled LC capacity from all LC contracts at time $t$ and the total realized (actually deployed) LC from all LC contracts at time $t$ in scenario $s$ is captured in (26). Equations in (27) describe the total capacity payment and total energy payment for all LC contracts at time $t$. Total payment for LC contracts at time $t$ in scenario $s$ is presented in (28), which also indicates that a LC contract $r$ at time $t$ can only be deployed if it is already scheduled in the day before the operating day.

8) Load Shifting: Similar to LC contracts, each LS contract $r$ includes similar information of prices $\left(c_{r, t}^{\mathrm{LS}, 1}, c_{r, t}^{\mathrm{LS}, 2}\right)$ and aggregated quantity $\left(q_{r, t}^{\mathrm{LS}}\right)$ in each time slot $t$. Additionally, each LS contract $r$ comprises the set of time slots $\left(L S P_{r, t}\right)$ to which the offered load in LS contract $r$ at time $t$ can be shifted. Hence, for $\forall r, t, s$, we have

$$
\begin{array}{r}
P_{t}^{\mathrm{LS}, 1}=\sum_{r=1}^{N S C} q_{r, t}^{\mathrm{LS}} U_{r, t}^{\mathrm{LS}}, \forall t ; \quad P_{t, s}^{\mathrm{LS}, 2}=\sum_{r=1}^{N L C} q_{r, t}^{\mathrm{LS}} u_{r, t, s}^{\mathrm{LS}} \\
C_{t}^{\mathrm{LS}, 1}=\sum_{r=1}^{\mathrm{NLS}} c_{r, t}^{\mathrm{LS}, 1} q_{r, r}^{\mathrm{LS}} U_{r, t}^{\mathrm{LS}} ; \quad C_{t, s}^{\mathrm{LS}, 2}=\sum_{r=1}^{\mathrm{NLS}} c_{r, t}^{\mathrm{LS}, 2} q_{r, t}^{\mathrm{LS}} u_{r, t, s}^{\mathrm{LS}} \\
C_{t, s}^{\mathrm{LS}}=C_{t}^{\mathrm{LS}, 1}+C_{t, s}^{\mathrm{LS}, 2} ; \quad u_{r, t, s}^{\mathrm{LS}} \leq U_{r, t}^{\mathrm{LS}} \\
U_{r, t}^{\mathrm{LS}}, u_{r, t, s}^{\mathrm{LS}} \in\{0,1\} ; \quad U_{r, t}^{\mathrm{LS}}=0, \quad \forall t \notin T_{r}^{\mathrm{LS}}
\end{array}
$$

where $T_{r}^{\mathrm{LS}}$ is the set of time slots during which LS contract $r$ offers a positive LS quantity. The constraints (29)-(32) for LS contracts are similar to those for LC contracts. Furthermore, for $\forall r, t, t^{\prime}, s$, we have following additional constraints

$$
\begin{array}{r}
v_{r, t, t^{\prime}, s}=0, \forall t^{\prime} \notin L R P_{r, t} \text { or } t^{\prime}=t \\
\sum_{t^{\prime} \in L R P_{r, t}} v_{r, t, t^{\prime}, s}=u_{r, t, s}^{\mathrm{LS}} ; \quad v_{r, t, t^{\prime}, s} \in\{0,1\} \\
P_{r, t, t^{\prime}, s}^{\mathrm{LR}}=q_{r, t}^{\mathrm{LS}} v_{r, t, t^{\prime}, s} ; \quad P_{r, t^{\prime}, s}^{\mathrm{LR}}=\sum_{t \in T_{r}^{\mathrm{LS}}} P_{r, t, t^{\prime}, s}^{\mathrm{LR}}
\end{array}
$$

where $v_{r, t, t^{\prime}, s}$ is an auxiliary binary variable, which is equal to " 1 " if the offered load in LS contract $r$ at time $t$ is deployed and recovered at time $t^{\prime}$ in scenario $s$, and $L R P_{r, t}$ is the set of time slots to which the offered load in LS contract $r$ at time $t$ can be shifted. Constraint (33) implies that the offered LS load in LC contracts cannot be shifted beyond the sets of time slots when the load can be recovered (i.e., sets $L R P_{r, t}$ ), and if a LS contract at time $t$ is deployed, the offered LS quantity must be shifted to a time slot which is different from $t$. Equation (34) states that if a LS contract $r$ is scheduled and deployed at time $t$ in scenario $s$ (i.e., $u_{r, t, s}^{\mathrm{LS}}=1$ ), the offered LS load must be shifted to a time slot in $L R P_{r, t}$. Obviously, if the load is not scheduled or is scheduled and not deployed (i.e., $u_{r, t, s}^{\mathrm{LS}}=0$ ), constraint (34) forces all $v_{r, t, t^{\prime}, s}$ to be zero. The first equation in (35) captures the amount of load recovered (i.e., $P_{r, t, t^{\prime}, s}^{\mathrm{LR}}$ ) from shifting load in contract $r$ at time $t$ to time $t^{\prime}$ in scenario $s$ while the other equation indicates the total load is shifted from contract $r$ to time $t^{\prime}$ in scenario $s$ (i.e., $P_{r, t^{\prime}, s}^{\mathrm{LR}}$ ). Finally, total load recovered at time $t^{\prime}$ from the deployment of all LS contracts is given as

$$
P_{t^{\prime}, s}^{\mathrm{LR}}=\sum_{r=1}^{N L S} P_{r, t^{\prime}, s}^{\mathrm{LR}}, \forall t^{\prime}, s .
$$

Remark. For simplicity, we assumed that the LC/LS offers in each time slot are independent, and if a $L C / L S$ contract at time $t$ (i.e., an offer) is scheduled and actually deployed, we assumed that the entire load in the offer must be curtailed or must be shifted to another (only one) time slot as in [8], [9]. However, the modeling of LC/LS contracts can be extended for other LC/LS models as in [6], [12]. For example, a LC/LS offer may allow to curtail/shift a portion instead of the entire offered load, or an offered LS quantity can be shifted/redistributed in several time slots instead of only one time slot, or some contracts may require a minimum and maximum LC/LS duration.

9) Power Balance: The sum of total power generation from DGs and WPs, and total net charging/discharging power from BESs, and the total LC, LS, and ILC is equal to the sum of the total load, the recovered load, and the actual power exchange with the grid. Hence,

$$
\begin{gathered}
\sum_{i=1}^{N G} P_{i, t, s}+\sum_{w=1}^{N W}\left(P_{w, t, s}-P_{w, t, s}^{\mathrm{ws}}\right)+P_{t, s}^{\mathrm{LC}, 2}+P_{t, s}^{\mathrm{LS}, 2}+D_{t, s}^{\mathrm{LLC}} \\
+\sum_{k=1}^{N K}\left(P_{k, t}^{s, \mathrm{~d}}-P_{k, t}^{s, \mathrm{c}}\right)=P_{t, s}^{\mathrm{del}}+D_{t, s}+P_{t, s}^{\mathrm{LR}}, \quad \forall s, t .
\end{gathered}
$$

It is worth mentioning that the total load at time $t$ in scenario $s$ is equal to $D_{t, s}-P_{t, s}^{\mathrm{LC}, 2}-D_{t, s}^{\mathrm{LLC}}-P_{t, s}^{\mathrm{LS}, 2}+P_{t, s}^{\mathrm{LR}}$.

\section{B. Risk-Constrained Stochastic Optimization Formulation}

Based on the concept of CVaR presented in Section II-C, we have the following risk-constrained optimization problem when the conditional value-at-risk at $\alpha$ confidence level ( $\alpha$ CVaR) is considered [24]

$$
\max \sum_{s=1}^{N S} \rho_{s} \text { Profit }_{s}+\beta\left\{\xi-\frac{1}{1-\alpha} \sum_{s=1}^{N S} \rho_{s} \eta_{s}\right\}
$$

subject to

$$
\begin{aligned}
\eta_{s}+\text { Profit }_{s}-\xi & \geq 0, \quad \forall s \\
\eta_{s} \geq 0, & \forall s
\end{aligned}
$$

and operation constraints $(11)-(37)$.

The profit in each scenario $s$ (i.e., Profit $t_{s}$ ) is given in (8) and constraints (39)-(40) are typical in CVaR-based formulation. This is indeed a mixed integer linear program (MILP), which can be solved by using CPLEX. 


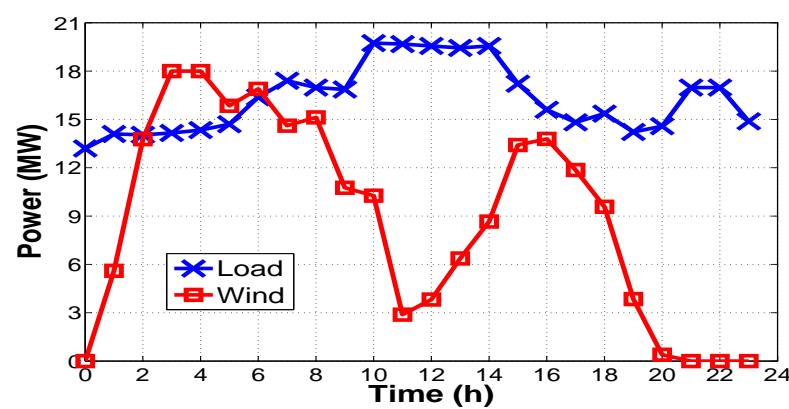

(a) Forecast wind power and load

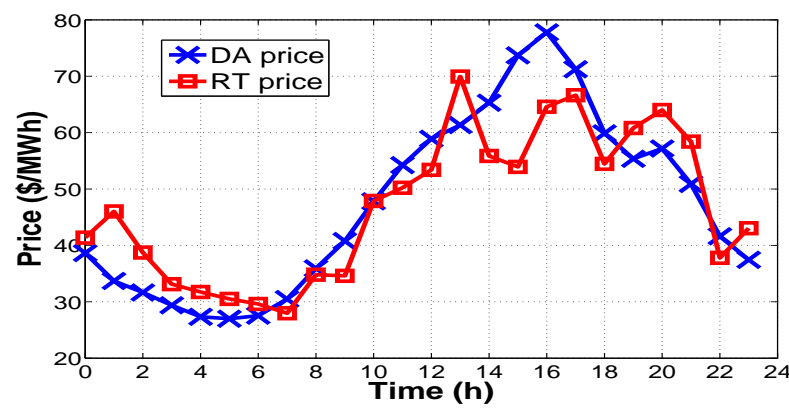

(b) Forecast DA and RT electricity prices

Fig. 1. Forecasts of wind power, load, and electricity prices

\section{Numerical Results}

We assume the scheduling horizon is one day which is divided into 24 equal time slots. We assume that the MG aggregator has a take-or-pay contract [13] to buy energy from a local wind farm, and wind energy price is fixed at $35 \$ / M W h$. The wind farm consists of 18 identical WPs where each WP has a rated power of $1 \mathrm{MW}$, and the cut-in, cut-out, and rated wind speeds are $3 \mathrm{~m} / \mathrm{s}, 30 \mathrm{~m} / \mathrm{s}$, and $12 \mathrm{~m} / \mathrm{s}$, respectively [45]. Additionally, the MG aggregator owns three DGs including two microturbines (MT) and one fuel cell (FC). Data for these DGs are taken from [45].

TABLE I

BATTERY PARAMETERS

\begin{tabular}{cccccc}
\hline \hline $\begin{array}{c}E^{\text {cap }} \\
(\mathrm{MWh})\end{array}$ & $S O C^{\text {min }}$ & $S O C^{\text {max }}$ & $S O C^{\text {ini }}$ & $\begin{array}{c}P^{\mathrm{c}, \max } \\
(\mathrm{MW})\end{array}$ & $\begin{array}{c}\mathrm{P}^{\mathrm{d}, \max } \\
(\mathrm{MW})\end{array}$ \\
\hline 5 & 0.3 & 0.9 & 0.5 & 1 & 1 \\
\hline
\end{tabular}

We assume that the MG aggregator possesses one BES. Parameters of the BES is presented in Table I. The initial SOC of the BES is assumed to be 0.5. The values of loss load and bid deviation penalty are set equal to $1000 \$ / \mathrm{MWh}$, and 5 \$/MWh [39], respectively. The maximum power exchange with the main grid is assumed to be $20 \mathrm{MW}$. Furthermore, in the base case, we assume the MG aggregator sells electricity to customers at a fixed retail price of $55 \$ / \mathrm{MWh}$. The value of confidence level parameter $\alpha$ and risk aversion parameter $\beta$ are set to 0.9 and 0.1 , respectively. Table II summarizes the system parameters in the base case. The system parameters in Table II are used to obtain all numerical results unless stated otherwise.

Data of LC and LS contracts is shown in Tables III and IV, respectively. For example, the LC contract 1 allows the MG
TABLE II

SYSTEM PARAMETERS IN THE BASE CASE

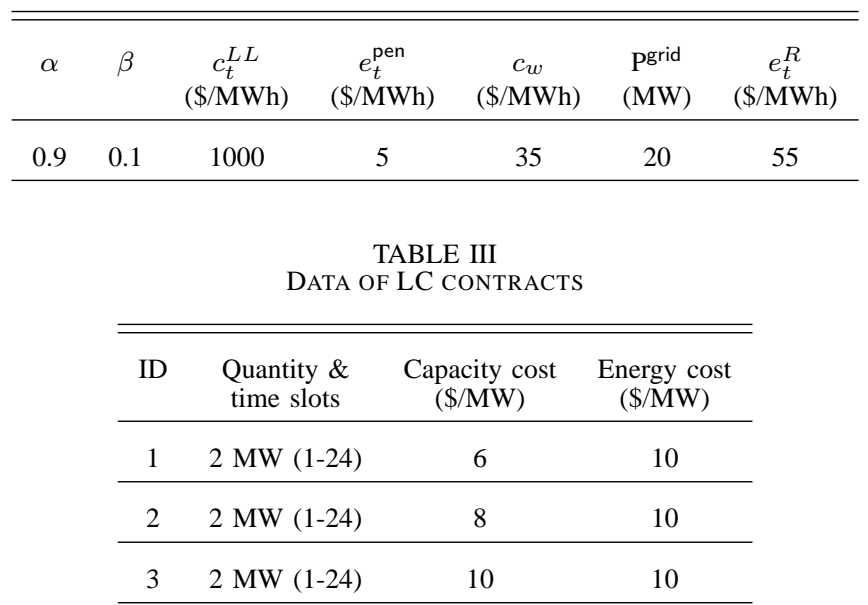

TABLE IV

DATA OF LS CONTRACTS

\begin{tabular}{llccc}
\hline \hline ID & $\begin{array}{l}\text { Quantity \& } \\
\text { time slots }\end{array}$ & $\begin{array}{c}\text { LR } \\
\text { periods }\end{array}$ & $\begin{array}{c}\text { Capacity cost } \\
(\$ / M W)\end{array}$ & $\begin{array}{c}\text { Energy cost } \\
(\$ / M W)\end{array}$ \\
\hline 1 & $\begin{array}{l}2 \text { MW (9-11, 20) } \\
3 \text { MW (15-19) }\end{array}$ & $5-22$ & 5 & 5 \\
\hline 2 & $\begin{array}{l}1 \text { MW (7, 8, 15) } \\
\text { 2 MW (9, 16) }\end{array}$ & $\begin{array}{l}4-10, \\
14-20\end{array}$ & 10 & 5 \\
& 3 MW (17-19) & & & \\
\hline 3 & $\begin{array}{l}\text { 2 MW (17-20) } \\
1 \text { MW (8-11) }\end{array}$ & $\begin{array}{l}6-12, \\
15-23\end{array}$ & 15 & 5 \\
\hline
\end{tabular}

aggregator to curtail maximum of $2 \mathrm{MW}$ of load for every hour in the operating day with the capacity price is $6 \$ / \mathrm{MW}$, and the energy price is $10 \$ / \mathrm{MW}$. The LS contract 3 allows the MG aggregator to shift $2 \mathrm{MW}$ of load at time slots $17-20$, and $1 \mathrm{MW}$ of load at time slots 8-11 to LR periods 6-12 and 15-23.

We use historical data of the uncertain parameters as their forecast values. The data of electricity prices is taken from [2]. Historical wind speed data is retrieved from [54]. Wind power curve is used to convert wind speed to wind power. The forecasts of total MG load, wind power generation, and electricity prices are presented in Figs. 1(a) and 1(b). Scenario generation and reduction are implemented as described in Section II-B. Due to the uncertainty in scenario generation and reduction, some in-sample tests and out-of-sample tests have been conducted to evaluate the performance of the tree construction method and verify the stability of the solution [24], [55]-[58]. We have applied the method in [55]-[57] to perform these stability tests. The tests suggest that 100 reduced scenarios are sufficient to achieve very reliable performance, which is, therefore, chosen to obtain all numerical results in this section. For brevity, these detailed stability studies are not presented in this paper.

The commitment statuses of LC and LS contracts for different values of retail rates are provided in Table V. We can observe that, for example, if the retail price is $50 \$ / \mathrm{MWh}, \mathrm{LC}$ contract 2 is scheduled at time slots $14,17,18$ to be possibly curtailed in the operating day. However, if the retail price is 55 
TABLE V

LC AND LS DAY AHEAD SCHEDULE

\begin{tabular}{ccccc}
\hline \hline Type & ID & $\begin{array}{c}\mathrm{e}_{t}^{R}=50 \\
(\$ / \mathrm{MWh})\end{array}$ & $\begin{array}{c}\mathrm{e}_{t}^{R}=55 \\
(\$ / \mathrm{MWh})\end{array}$ & $\begin{array}{c}\mathrm{e}_{t}^{R}=60 \\
(\$ / \mathrm{MWh})\end{array}$ \\
\hline LC & 1 & $14,17,18$ & $14,17,18$ & $\mathrm{X}$ \\
LC & 2 & $14,17,18$ & 17,18 & $\mathrm{X}$ \\
LC & 3 & 17,18 & $\mathrm{X}$ & $\mathrm{X}$ \\
\hline LS & 1 & $9-11,15-20$ & $9-11,15-20$ & $9-11,15-20$ \\
LS & 2 & $15-19$ & $15-19$ & $15-19$ \\
LS & 3 & $11,17-20$ & $11,17-20$ & $11,17-20$ \\
\hline
\end{tabular}

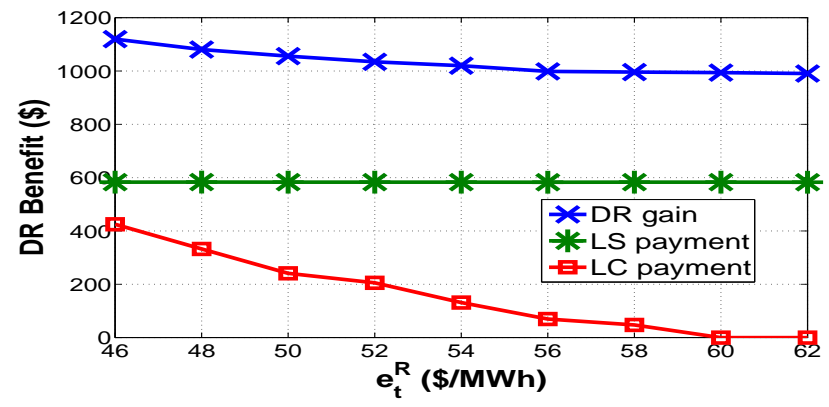

Fig. 2. Impact of retail price on the optimal solution

\$MWh, LC contract 2 is only scheduled at time slots 17,18 , and LC contract 2 is not scheduled at all for the operating day if the retail price is $60 \$ / M W h$. Similar observations can be drawn for LC contract 1 and 3 . When the retail price is high, LC contracts may not be scheduled at all. However, in reality, it is desirable that MG aggregator or any load serving entity offers the retail price as low as possible to be competitive in the market. Otherwise, customers can switch to other energy providers [24]. We can see that LS contract 1 is scheduled at all the time slots $(10,11,15-20)$ that it offered LS options to the MG aggregator. However, LS contract 2 is scheduled only at time slots 15-19, and its shiftable loads at time slots 7-9 are not scheduled. Similar to LS contract 2, LS contract 3 is not scheduled at several time slots (8-10) that it offered LS options to the MG aggregator.

Fig. 2 shows the impact of retail price on the optimal solution. DR gain is defined as the difference between the optimal expected profit when DR contracts are integrated into the optimization framework of the MG aggregator and the optimal expected profit when there is no DR considered. LC and LS payments are the expected values (i.e., sum of capacity and energy payments) that the MG aggregator pays to customers or the intermediate agent that collects customers' DR aggregation. The LC payment indeed decreases as the retail price increases. The LC payment tends to zero if the retail price approaches $60 \$ / \mathrm{MWh}$. In fact, there is no LC contract scheduled when the retail price is sufficiently large. However, LS does not change the total load; therefore, it does not affect the retail revenue of the MG aggregator. The scheduling and deployment of LS contracts depends mostly on the DA and RT prices. Therefore, it is almost constant as the retail price is varied. The DR gain reduces as the retail price increases since the benefit of LC contracts diminishes.

Figs. 3(a) and 3(b) illustrate how the price and quantity in LC and LS contracts impact the attained benefits of DR. We

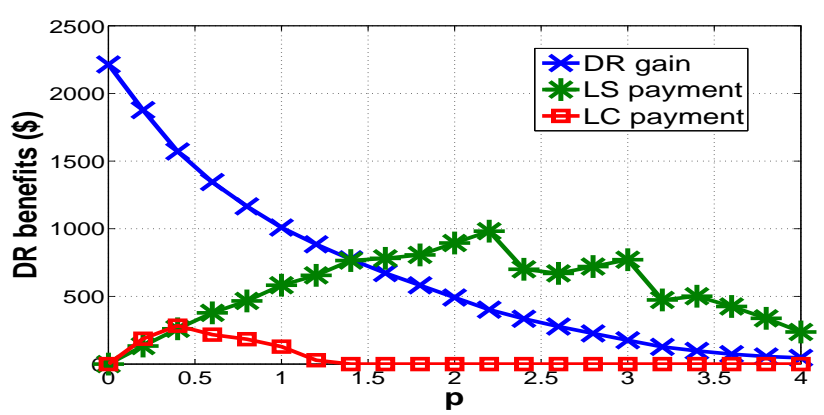

(a) Varying DR price

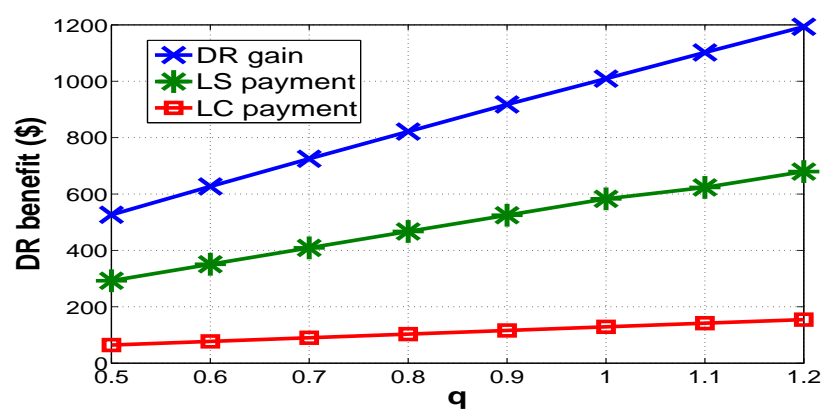

(b) Varying quantity of DR load

Fig. 3. Impact of DR offers on the optimal solution

have defined a scaling factor $p$ based on which we scale the prices in Tables III and IV in this study. In particular, it is more expensive to utilize LC/LS contracts as $p$ increases. As evident in Fig. 3(a), the LC contracts are only utilized when the DR price is small. Also, the LS contracts tend to be scheduled less frequently (in terms of scheduled quantity of load in LS contracts) as the DR price increases. As a result, the DR gain decreases as $p$ increases. We have also defined another scaling factor $q$ that we use to scale all load quantities in Tables III and IV. As expected, the DR gain increases, and the payment to customers increases as $q$ increases. In other words, both the MG aggregator and MG customers enjoy more benefits as larger DR load is offered.

The impacts of risk aversion parameter $\beta$ on the optimal solution is shown in Figs. 4(a)-4(d), 5(a) and 5(b). As the level of risk-aversion of the MG aggregator increases, it is expected that the CVaR increases and the expected profit of the MG aggregator decreases [24], [30]. This is confirmed by the results in Figs. 4(a)-4(d), 5(a) and 5(b). Furthermore, Figs. 5(a) and 5(b) illustrate the benefit attained by using battery storage and the proposed DR contract scheme. The expected profit of the MG aggregator is higher when a battery storage facility is utilized with parameters given in Table I compared to the case where the aggregator does not own any BESs. Furthermore, integrating the proposed DR scheme into the operation optimization of the MG aggregator results in better profit compared to the case where DR is not considered.

Finally, Figs. 6(a)-6(c) show the hourly DA power bid quantity that the MG aggregator submits to the DA market for different values of risk aversion parameter $\beta$. In our proposed bidding model, which is applicable to the US market [39]-[41], the aggregator has the opportunity to arbitrate the difference between the DA price and the RT price. In fact, if the DA price is smaller than the RT price at a particular time 


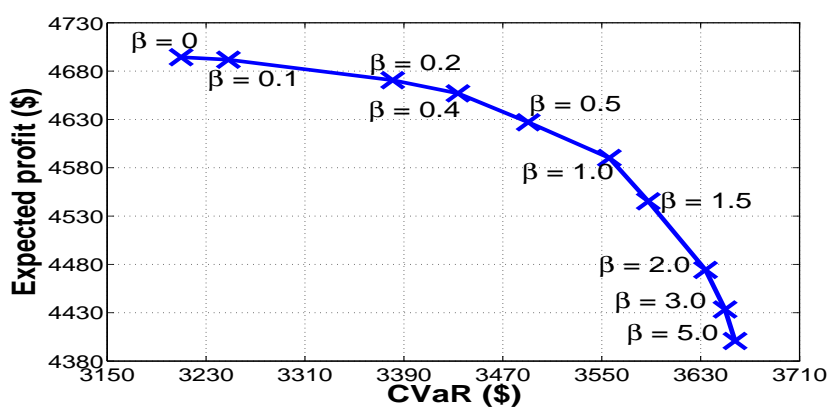

(a) $e_{t}^{R}=55 \$ / \mathrm{MWh}$, with DR

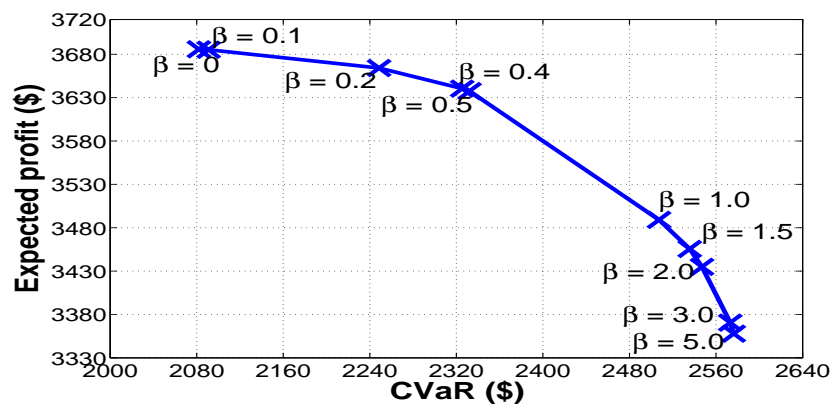

(b) $e_{t}^{R}=55 \$ / \mathrm{MWh}$, no DR

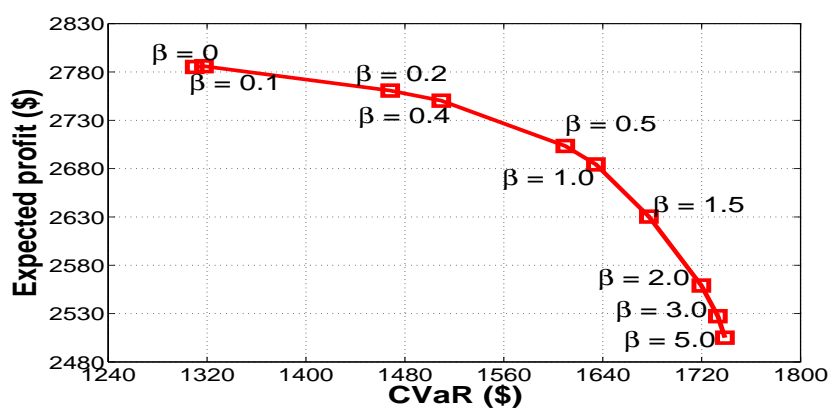

(c) $e_{t}^{R}=50 \$ / \mathrm{MWh}$, with DR

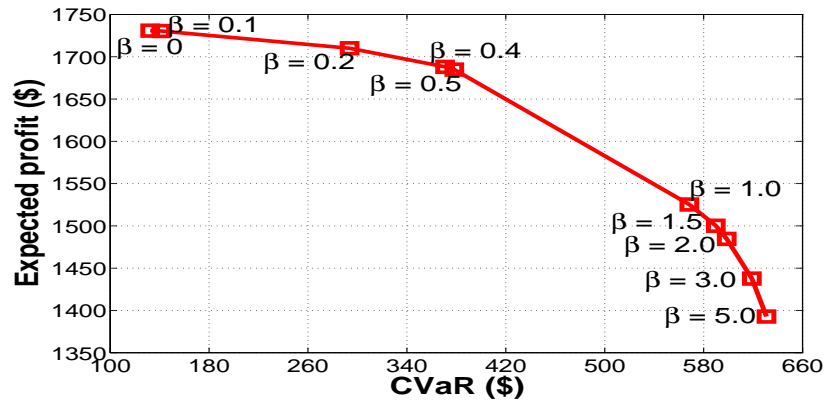

(d) $e_{t}^{R}=50 \$ / \mathrm{MWh}$, no DR

Fig. 4. Impact of risk aversion parameter $\beta$ on the optimal solution

slot, the MG aggreagtor can make profit by bidding an excess power beyond what needed to support its load, then resell a portion to the RT market to take the advantage of the price difference. Note that a penalty may be applied when the actual delivery differs from the DA schedule.

As can be observed, by integrating the proposed DR scheme to the bidding decision making process, the MG aggregator tends to buy more power in the DA market during time slots when the expected DA price is smaller than the RT price (e.g.,

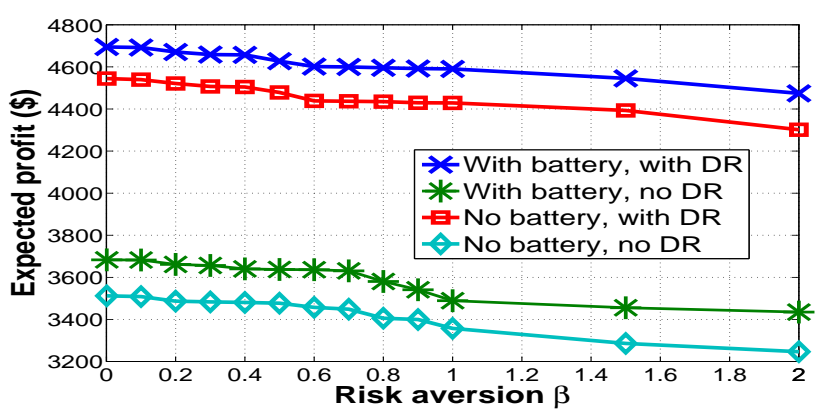

(a) Expected Profit

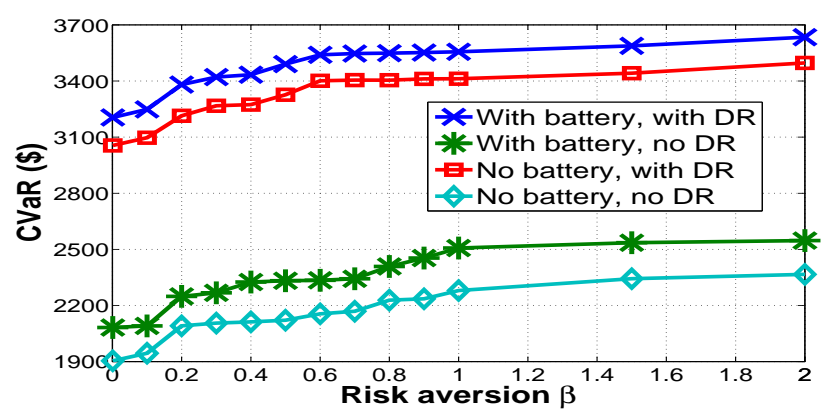

(b) CVaR

Fig. 5. Impact of risk aversion $\beta$, battery, and DR on the optimal solution

time slots 3-6), and it tends to sell more power in the DA market during the time slots when the expected DA price is greater than the expected RT price (e.g., time slots 1618). Consequently, the MG aggregator can make more profit when DR is considered compared to the case where DR is not considered. Furthermore, since the RT forecast price tends to contain more uncertainties than the DA forecast price [41], in several realized scenarios the RT price is higher than the DA price even the forecast RT price is lower than the forecast DA price shown in Fig. 1(b). Hence, when the forecast DA price is larger than the forecast RT price (e.g. 14-18), for both cases with and without DR, the aggregator would sell less power in the DA market as the risk aversion parameter $\beta$ increases to reduce the risk of achieving low profits in some bad scenarios.

\section{CONClusion ANd Future Work}

We have proposed a framework for optimizing energy trading operations of a MG aggregator in the DA energy market. Uncertainties in the system load, renewable energy generation, and electricity prices have been tackled by employing the scenario-based Monte Carlo simulation technique. The tradeoff between maximizing the expected profit of the MG aggregator and the risk of getting low profits of the MG aggregator in bad scenarios has been modeled by using the CVaR approach. Furthermore, we have proposed to integrate the concept of DR contract between the MG aggregator and customers into the framework. Numerical results have confirmed that the deployment of LC and LS contracts provides improved benefits for both MG aggregator and customers.

There are several directions that the proposed framework can be further extended. First, the proposed framework can be modified and adopted by various market entities such as a MG aggregator, a retailer, a DISCO, or a LSE. However, the scale of the considered system must be sufficiently large so 


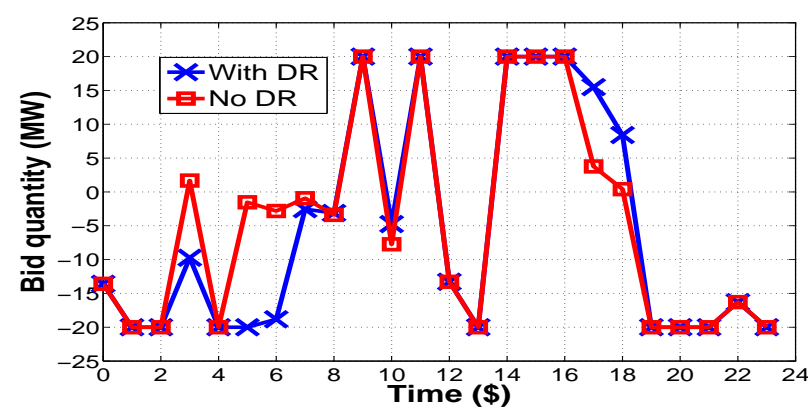

(a) $\beta=0$

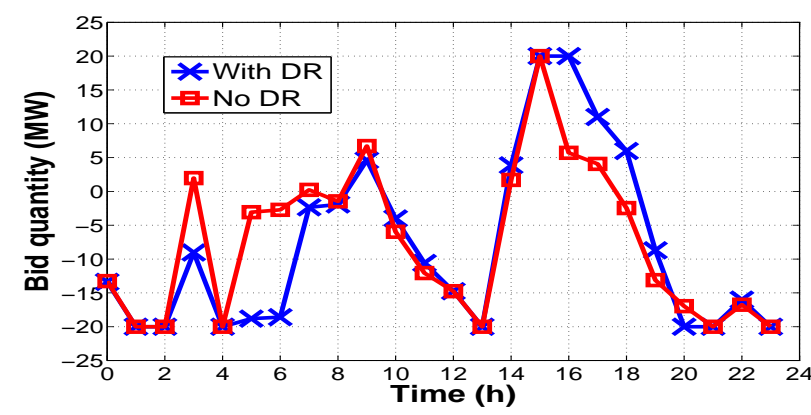

(b) $\beta=1$

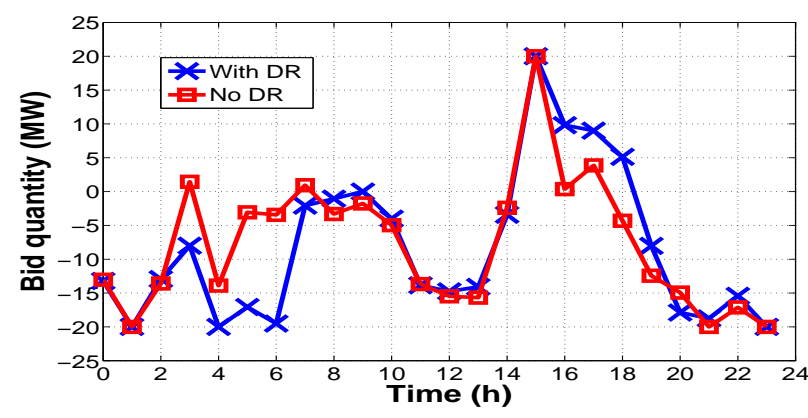

(c) $\beta=5$

Fig. 6. Hourly DA bid quantity for different values of $\beta$

that the underlying entity (e.g., a MG aggregator) is allowed to participate in the wholesale market. The system size must also be large to make DR contracts meaningful and profitable to customers. In addition, customers' base load should be substantial so that it can make significant and stable DR offers. Therefore, modification of the proposed model for medium or small scale MGs would be an interesting research topic for further study. Second, despite the fact that there are many types of DR contracts that the MG aggregator can offer to customers, it is difficult for customers to choose the best offer price to maximize their benefits. This is because if customers offer a high price, their DR contracts may not be scheduled by the MG aggregator; however, low DR prices may not provide customers sufficient motivation to participate in these DR programs. Moreover, the MG aggregator may also want to design a price which not only maximizes its profit but also maximizes the amount of DR quantities from customers. Designing a novel pricing structure for DR contracts is an interesting and open research topic. Finally, advanced forecasting techniques as well as modeling the correlation among system uncertainties need further study to fully understand their impacts on the optimal solutions.

\section{REFERENCES}

[1] The Federal Energy Regulatory Commission's Order 745: www.ferc.gov/EventCalendar/Files/20110315105757-RM10-17-000.pdf

[2] http://pjm.com/markets-and-operations.aspx

[3] http://www.nyiso.com/public/markets_operations

[4] A. Khodaei, M. Shahidehpour, and S. Bahramirad, "SCUC with hourly demand response considering intertemporal load characteristics," IEEE Trans. Smart Grid, vol. 2, no. 3, pp. 564-571, Sep. 2011.

[5] H. Wu, M. Shahidehpour, and M.E. Khodayar, "Hourly demand response in day-ahead scheduling considering generating unit ramping cost," IEEE Trans. Power Syst., vol. 28, no. 3, pp. 2446-2454, Aug. 2013.

[6] M. Parvania and M. Fotuhi-Firuzabad, "Integrating load reduction into wholesale energy market with application to wind power integration," IEEE Trans. Syst. J., vol. 6, no. 1, pp. 35-45, Mar. 2012.

[7] A. Yousefi, H.H.C. Iu, T. Fernando, and H. Trinh, "An approach for wind power integration using demand side resources," IEEE Trans. Sustain. Energy, vol. 4, no. 4, pp. 917-924, Oct. 2013.

[8] M. Parvania and M. Fotuhi-Firuzabad, "Demand response scheduling by stochastic SCUC," IEEE Trans. Smart Grid, vol. 1, no. 1, pp. 89-98, Jun. 2010.

[9] C. Sahin, M. Shahidehpour, and I. Erkmen, "Allocation of hourly reserve versus demand response for security-constrained scheduling of stochastic wind energy," IEEE Trans. Sustain. Energy, vol. 4, no. 1, pp. 219-228, Jan. 2013.

[10] A.J. Conejo, J.M. Morales, and L. Baringo,"Real-time demand response model," IEEE Trans. Smart Grid, vol. 1, no. 3, pp. 236-242, Dec. 2010.

[11] Z. Chen, L. Wu, and Y. Fu, "Real-time price-based demand response management for residential appliances via stochastic optimization and robust optimization," IEEE Trans. Smart Grid, vol. 3, no. 4, pp. 1822 1831, Dec. 2012.

[12] M. Parvania, M. Fotuhi-Firuzabad, and M. Shahidehpour, "Optimal demand response aggregation in wholesale electricity markets," IEEE Trans. Smart Grid, vol. 4, no. 4, pp. 1957-1965, Dec. 2013.

[13] M. Rahimiyan, L. Baringo, and A.J. Conejo, "Energy management of a cluster of interconnected price-responsive demands," IEEE Trans. Power Syst., vol. 29, no. 2, pp. 645-655, Mar. 2014.

[14] D.T. Nguyen and L.B. Le, "Optimal bidding strategy for microgrids considering renewable energy and building thermal dynamics," IEEE Trans. Smart Grid, vol. 5, no. 4, pp. 1608-1620, Jul. 2014.

[15] B. Zhao, Y. Shi, X. Dong, W. Luan, J. Bornemann, "Short-term operation scheduling in renewable-powered microgrids: a duality-based approach," IEEE Trans. Sustain. Energy, vol. 5, no. 1, pp. 209-217, Jan. 2014.

[16] R.P. Behnke, C. Benavides, F. Lanas, B. Severino, L. Reyes, J. Llanos, and D. Saez, "A microgrid energy management system based on the rolling horizon strategy," IEEE Trans. Smart Grid, vol. 4, no. 2, pp. 996-1006, Jun. 2013.

[17] X. Guan, Z. Xu, Q.S. Jia, "Energy-efficient buildings facilitated by microgrid," IEEE Trans. Smart Grid, vol. 1, no. 3, pp. 243-252, Dec. 2010.

[18] T. Wu, Q. Yang, Z. Bao, and W. Yan, "Coordinated energy dispatching in microgrid with wind power generation and plug-in electric vehicles," IEEE Trans. Smart Grid, vol. 4, no. 3, pp. 1453-1463, Sept. 2013.

[19] D.T. Nguyen and L.B. Le, "Optimal energy management for cooperative microgrids with renewable energy resources," in Proc. IEEE SmartGridComm, Vancouver, Oct. 2013.

[20] D.T. Nguyen and L.B. Le, "Optimal energy trading for building microgrid with electric vehicles and renewable energy resources," in Proc. IEEE PES Innov. Smart Grid Technol. (ISGT'2014), Washington, DC, Feb. 2014

[21] A.G. Tsikalakis and N.D. Hatziargyriou, "Centralized control for optimizing microgrids operation," IEEE Trans. Energy Convers., vol. 23, no. 1, pp. 241-248, Mar. 2008.

[22] A. Khodaei and M. Shahidehpour, "Microgrid-based co-optimization of generation and transmission planning in power systems," IEEE Trans. Power Syst., vol. 28, no. 2, pp. 1582-1590, May 2013.

[23] R. T. Rockafellar and S. Uryasev, "Conditional value-at-risk for general loss distributions," J. Bank. Fin., vol. 26, no. 7, pp. 14431471, Jul. 2002.

[24] A. J. Conejo, M. Carrion, and J. M. Morales, Decision Making Under Uncertainty in Electricity Markets, International Series in Operations Research Management Science, Springer, New York. 2010.

[25] M. Sadeghi and S. Shavvalpour, "Energy risk management and value at risk modeling," Energy Policy, vol. 34, no. 18, pp. 3367-3373, Dec. 2006.

[26] M. Carrion, A.J. Conejo, and J.M. Arroyo, "Forward contracting and selling price determination for a retailer," IEEE Trans. Power Syst., vol. 22, no. 4, pp. 2105-2114, Nov. 2007.

[27] R. Garcia-Bertrand, "Sale prices setting tool for retailers," IEEE Trans. Smart Grid, vol. 4, no. 4, pp. 2028-2035, Dec. 2013. 
[28] M. Carrion, J.M. Arroyo, A.J. Conejo, "A bilevel stochastic programming approach for retailer futures market trading," IEEE Trans. Power Syst., vol. 24, no. 3, pp. 1446-1456, Aug. 2009.

[29] A.J. Conejo, R. Garcia-Bertrand, M. Carrion, A. Caballero, and A. de Andres, "Optimal involvement in futures markets of a power producer," IEEE Trans. Power Syst., vol. 23, no. 2, pp. 703-711, May 2008.

[30] J.M. Morales, A.J. Conejo, and J. Perez-Ruiz, "Short-term trading for a wind power producer," IEEE Trans. Power Syst., vol. 25, no. 1, pp. 554-564, Feb. 2010.

[31] J.P.S. Catalao, H.M.I. Pousinho, and V.M.F. Mendes, "Optimal offering strategies for wind power producers considering uncertainty and risk," IEEE Syst. J., vol. 6, no. 2, pp. 270-277, Jun. 2012.

[32] L. Baringo and A.J. Conejo, "Risk-constrained multi-stage wind power investment," IEEE Trans. Power Syst., vol. 28, no. 1, pp. 401-411, Feb. 2013.

[33] A. Safdarian, M. Fotuhi-Firuzabad, and M. Lehtonen, "A stochastic framework for short-term operation of a distribution company," IEEE Trans. Power Syst., vol. 28, no. 4, pp. 4712-4721, Nov. 2013.

[34] M. Carrion, A.B. Philpott, A.J. Conejo, and J.M. Arroyo, "A stochastic programming approach to electric energy procurement for large consumers," IEEE Trans. Power Syst., vol. 22, no. 2, pp. 744-754, May 2007.

[35] G. Lu, F. Wen, C.Y. Chung, and K.P. Wong, "Conditional value-at-risk based mid-term generation operation planning in electricity market environment," in Proc. IEEE Congr. Evolutionary Computation, Singapore, 2007.

[36] Y. Zhang and G. B. Giannakis, "Robust optimal power flow with wind integration using conditional value-at-risk," in Proc. IEEE SmartGridComm, Vancouver, Oct. 2013.

[37] A.T. Al-Awami and M.A. El-Sharkawi, "Coordinated trading of wind and thermal energy," IEEE Trans. Sustain. Energy, vol. 2, no. 3, pp. 277-287, Jul. 2011.

[38] A.A. Sanchez de la Nieta, J. Contreras, and J.I. Munoz, "Optimal coordinated wind-hydro bidding strategies in day-ahead markets," IEEE Trans. Power Syst., vol. 28, no. 2, pp. 798-809, May 2013.

[39] A. Botterud, Z. Zhou, J. Wang, R.J. Bessa, H. Keko, J. Sumaili, and V. Miranda, "Wind power trading under uncertainty in LMP markets," IEEE Trans. Power Syst., vol. 27, no. 2, pp. 894-903, May 2012.

[40] M. E. Khodayar and M. Shahidehpour, "Stochastic price-based coordination of intrahour wind energy and storage in a generation company," IEEE Trans. Sustain. Energy, vol. 4, no. 3, pp. 554-562, Jul. 2013.

[41] Q. Wang, J. Wang, and Y. Guan, "Price-based unit commitment with wind power utilization constraints," IEEE Trans. Power Syst., vol. 28, no. 3, pp. 2718-2726, Aug. 2013.

[42] R. Herranz, A.M. San Roque, J. Villar, and F.A. Campos, "Optimal demand-side bidding strategies in electricity spot markets," IEEE Trans. Power Syst., vol. 27, no. 3, pp. 1204-1213, Aug. 2012.

[43] J. Wang, M. Shahidehpour, L. Zuyi, "Security-constrained unit commitment with volatile wind power generation," IEEE Trans. Power Syst., vol. 23, no. 3, pp. 1319-1327, Aug. 2008.

[44] Y. Chen, J. Wen, and S. Cheng, "Probabilistic load flow method based on Nataf transformation and latin hypercube sampling," IEEE Trans. Sustain. Energy, vol. 4, no. 2, pp. 294-301, Apr. 2013.

[45] S. X. Chen, H. B. Gooi, and M. Q. Wang, "Sizing of energy storage for microgrids," IEEE Trans. Smart Grid, vol. 1, no. 4, pp. 1997-2006, Mar. 2012.

[46] J. Wang, S.W. Kennedy, and J.L. Kirtley, "Optimization of forward electricity markets considering wind generation and demand response," IEEE Trans. Smart Grid, vol. 5, no. 3, pp. 1254-1261, May 2014.

[47] J. Dupacová, N. Growe-Kuska, and W. Romisch, "Scenario reduction in stochastic programming: An approach using probability metrics," Math. Program, pp. 493-511, 2003.

[48] H. Heitsch and W. Romisch, "Scenario reduction algorithms in stochastic programming," Comput. Optimiz. Appl., vol. 24, pp. 187-206, Feb. 2003.
[49] J.M. Morale, "Impact on system economics and security of a high penetration of wind power," PhD Thesis, Universidad de Castilla-la Mancha, 2010.

[50] http://www.gams.com/docs/document.htm

[51] GAMS/SCENRED Documentation [online]. Available: http://www.gams.com/dd/docs/solvers/scenred2.pdf

[52] M. Carrion, and J. M. Arroyo, "A computationally efficient mixedinteger linear formulation for the thermal unit commitment problem," IEEE Trans. Power Syst., vol. 21, no. 3, pp.1371-1378, Aug. 2006.

[53] H. Pandzic, J.M. Morales, A.J. Conejo, and I. Kuzle, "Offering model for a virtual power plant based on stochastic programming," J. Applied Energy, vol. 105, pp. 282-292, May 2013.

[54] http://www.nrel.gov/midc/nwtc_m2/

[55] M. Kaut and S.W. Wallace, "Evaluation of scenario generation methods for stochastic programming," Pac. J. Optimiz., vol. 3, no. 2, pp. 257271, 2007.

[56] A.J. King and S.W. Wallace, "Modeling with Stochastic Programming," Springer, 2012.

[57] K. Bruninx, E. Delarue, and W. Dhaeseleer, "A modeling framework for the integration of intermittent renewables: stochastic unit commitment," Working Paper, 2014. Available [Online]: http://www.mech.kuleuven.be/en/tme/research.

[58] G. Bayraksan and D. P. Morton, "Assessing solution quality in stochastic programs via sampling," Tutorials in Operations Research, M.R. Oskoorouchi (ed.), 102-122, INFORMS 2009.

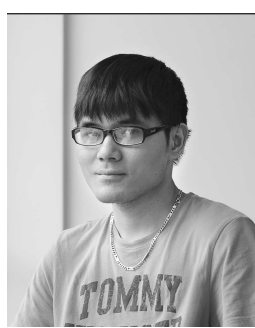

Duong Tung Nguyen received the B.Eng. from Hanoi University of Technology, Vietnam, in 2011 $\mathrm{He}$ is currently a graduate student at the Institut $\mathrm{Na}$ tional de la Recherche Scientifique (INRS), Université du Québec, Montréal, QC, Canada. His research interests lie at the intersection of operations research, economics, and engineering with current focus on control and optimization issues for microgrids.

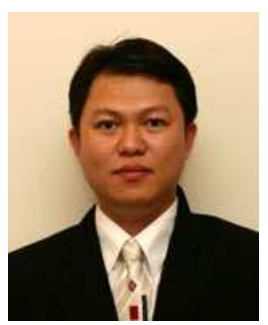

Long Bao Le (S'04-M'07-SM'12) received the B.Eng. (with Highest Distinction) degree from Ho Chi Minh City University of Technology, Vietnam, in 1999, the M.Eng. degree from Asian Institute of Technology, Pathumthani, Thailand, in 2002, and the Ph.D. degree from the University of Manitoba, Winnipeg, MB, Canada, in 2007.

He was a postdoctoral researcher at Massachusetts Institute of Technology (2008-2010) and University of Waterloo (2007-2008). Since 2010, he has been an assistant professor with the Institut National de la Recherche Scientifique (INRS), Université du Québec, Montréal, QC, Canada. His current research interests include smartgrids, cognitive radio and dynamic spectrum sharing, radio resource management, network control and optimization for wireless networks. He is a co-author of the book Radio Resource Management in Multi-Tier Cellular Wireless Networks (Wiley, 2013). Dr. Le is a member of the editorial board of IEEE Communications Surveys and Tutorials and IEEE Wireless Communications Letters. He has served as technical program committee co-chairs of the Wireless Access track at IEEE VTC2014-Fall, Wireless Networks track at IEEE VTC2011-Fall, and the Cognitive Radio and Spectrum Management track at IEEE PIMRC2011. 